\title{
History of HAART - the true story of how effective multi-drug therapy was developed for treatment of HIV disease Martin Delaney*
}

\author{
Address: Founder and Director, Project Inform, San Francisco, CA 94103, USA
}

* Corresponding author

from 2006 International Meeting of The Institute of Human Virology

Baltimore, USA. 17-2I November, 2006

Published: 2I December 2006

Retrovirology 2006, 3(Suppl I):S6 doi: I0.I I86/1742-4690-3-SI-S6

(C) 2006 Delaney; licensee BioMed Central Ltd.

The development of multi-drug combination therapy for treatment of HIV disease is considered one of the great success stories of modern medicine. In a period of approximately ten years, the death rate from HIV disease was reduced by 50 to $80 \%$ and changed from a nearly universally fatal and catastrophic illness to what is now often a manageable chronic illness. The story of how this was accomplished, however, is less well known than the outcome itself, which has been widely heralded in the medical and public media. The story of this accomplished though has often been widely distorted in the popular press and varies considerably from one country to the next. The media, in its usual quest for simple explanations, suggests that the development of multi-drug combination therapy was a new and unique discovery that could largely be attributed to a single research team. The true story is much more complex and acknowledges the contributions of a wide range of scientists in both academia and the private pharmaceutical industry. This presentation traces the historical basis which lead to the development of the individual therapies and the models of combination therapy long employed against other diseases. Rather than being the work of any single group, the development of HAART is seen to be attributed to a long string of discoveries by multiple groups and individuals beginning in the 1970s, built upon in the 1980s, and brought to their ultimate fruition in the 1990s. Major contributions came from people working in basic science, bio-chemistry, drug development and clinical testing in dozens of institutions and companies. It was only through these collective contributions that the success heralded in the mid-1990s came to be, and it s only through similar, continuing con- tributions that patients today are benefiting from the potency, durability and ease of use associated with today's best regimens. It is a disservice to science, however, to suggest, as the media has done, that any individual or group was primarily or most responsible for these advances. Instead, they are the work and accomplishment of the entire field of HIV medicine. 